\title{
PHOTOELECTRON BEAMS FROM THE UCLA RF GUN*
}

\author{
S. Park, N. Barov, S. Hartman, C. Pellegrini, J. Rosenzweig \\ P. Tran, G. Travish, R. Zhang \\ Department of Physics, University of California, Los Angeles, CA 90024 \\ P. Davis, G. Hairapetian, C. Joshi, N. Luhmann Jr. \\ Department of Electrical Engineering \\ University of California, Los Angeles, CA 90024
}

\begin{abstract}
A high brightness, low emittance photocathode rf gun is starting operation at UCLA as an injector to a $20 \mathrm{MeV}$ linac. This linac will initially be used to drive FELs, plasma wakefield accelerators, and to test plasma lenses. The gun is a $1 \frac{1}{2}$ cell $\pi$-mode standing wave structure running at $2.856 \mathrm{GHz}$, and has a copper photocathode. In the initial commissioning of the gun, photoelectron beams of up to $2.5 \mathrm{nC}$ at $4.5 \mathrm{MeV}$ have been produced. We report on the current status of the system, experimental data taken with 50 ps UV laser pulses, and plans for the future.
\end{abstract}

\section{INTRODUCTION}

In this paper we describe the status of the $20 \mathrm{MeV}$ high brightness, compact linear accelerator at UCLA. The $\sim 4 \mathrm{MeV}$ photoinjector gun which will be used to drive a linac system consists of a photocathode $\mathrm{rf}$ gun producing a $4 \mathrm{MeV}$ beam, followed by an accelerating structure to raise the energy to about $20 \mathrm{MeV}$. The beam from the linac can be sent in two beam lines, where experiments utilizing this beam can be performed. For initial measurements, a simplified beam line has been set up (Fig. 1). The experimental program is oriented towards: the development of high brightness beams from photocathodes and rf guns; plasma acceleration and focusing, FELs and other areas $[1,2]$ where small emittance and high brightness are important.

An $\mathrm{rf}$ gun with a photocathode is thought to have lower emittance and higher brightness, compared to the more traditional thermionic guns. The lower emittance is due to higher acceleration gradient and optimal phasing of the laser with respect to the rf pulses. The high brightness arises from high charge extraction in a short bunch length, as well as low emittance. Ideally, the spatial and temporal distribution of the photoelectrons at the cathode should be entirely determined by the laser beam profile impinging upon the cathode, so that the electron beam can be completely shaped by means of compression, focusing, and polarization of the laser beam.

In this paper we will describe the characteristics and the present status of the rf gun, the laser illuminating the photocathode, the rf system and controls, the beam line and diagnostics. We will then present some initial measurements 

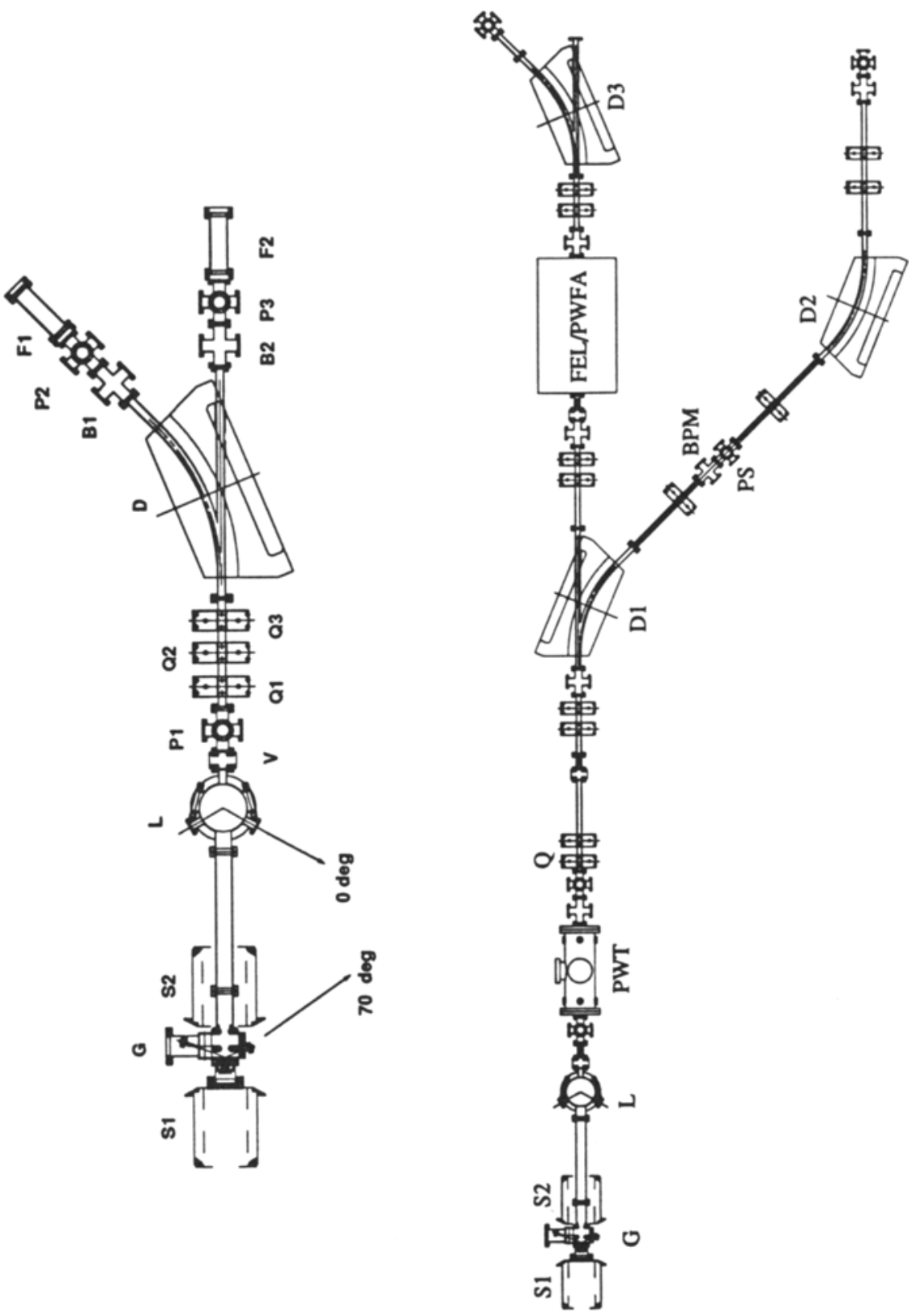

Figure 1: Setup for the quantum efficiency measurements (left) and plan for the future expansion (right). Some components shown here are: $\operatorname{gun}(\mathrm{G})$, laser coupling box(L), solenoid(S), dipole(D), quadrupole(Q), BPM(B), phosphor screen(P or PS), and Faraday cup(F). The FEL/PWFA box in the right figure represents the location of the free electron laser or a plasma wakefield accelerator experiment. 
on the photoelectron beam produced by the gun when approximately $50 \mathrm{ps}$ long laser pulse irradiated the photocathode.

\section{PHOTOCATHODE RF GUN}

The gun design is based on the Brookhaven[3] approach. Optimal coupling of rf power to the cavity, balancing of electric field between the half cell and full cell, and frequency tuning are facilitated by adjustment of cathode position, tuner and rf probe at each cell. The cathode position is controlled through a micrometer and can be finely adjusted. The $Q$ values of the two cells can be determined from the decay of the rf electric field following turning off of the rf. These results show that in the present configuration there is an unbalance between the two cells as seen in Figs. 2(a) and 2(b), which may indicate degradation of the rf coupling to the gun.

The gun can be initially tuned at atmospheric pressure. Once it is installed in the system and pumped down, atmospheric pressure deforms the cavity, resulting in detuning. This is compensated for by passing temperature controlled water through cooling channels of the gun. The rf power reflected from the gun is measured as a function of the gun temperature. With $20 \mathrm{~dB}$ attenuation, the input power to the gun is about $100 \mathrm{~kW}$. This level of power is too low for radiation to be of concern, yet sufficiently high for detection through a $50 \mathrm{~dB}$ waveguide directional coupler as shown in Fig. 2(c). For minimum reflection, which corresponds to resonance, the temperature is set.

The photocathode is machined from a solid block of OFHC copper to a tolerance of less than 0.001 inch in surface roughness. No active polishing was done. It was baked in situ with the temperature no higher than $100^{\circ} \mathrm{C}$ for about three days. When the cathode is exposed to air for a few hours for beamline modification, conditioning by $5 \mathrm{MW}$ rf and UV laser pulses of up to $200 \mu \mathrm{J}$ for a day at 1 $\mathrm{Hz}$ seems to restore the surface for photoemission.

\section{LASER AND OTHER OPTICS}

The laser system starts with a mode locked Coherent Antares Nd:YAG laser at $1064 \mathrm{~nm}$ wavelength. A crystal oscillator at $38.08 \mathrm{MHz}$ drives both the mode locker and gives a seed signal to be multiplied by 75 times to $2.856 \mathrm{GHz}$ for the $\mathrm{RF}$ system. The beam at $1064 \mathrm{~nm}$ is passed through a $2.2 \mathrm{~km}$ optical fiber via self phase modulation for spectral broadening and linearization of the chirp via group velocity dispersion before it enters a Continuum $\mathrm{Nd}$ :Glass regenerative amplifier, which is pulsed at $5 \mathrm{~Hz}$ by Stanford Research DG-535 pulser, which triggers the $\mathrm{RF}$ system simultaneously. The beam from the amplifier can be compressed to less than 4 ps by a grating pair and frequency doubled twice to a wavelength of 266 $\mathrm{nm}$ by two KD*P (potassium dihydrogen phosphate) crystals. This UV beam is transported through a maze of radiation shielding and then to the cathode, either via a window at the gun for a $70^{\circ}$ angle of incidence or via the laser coupling box for a $2.5^{\circ}$ angle of incidence. All the windows for the UV beam passage are made of quartz. 
634 Photoelectron Beams from the UCLA RF Gun
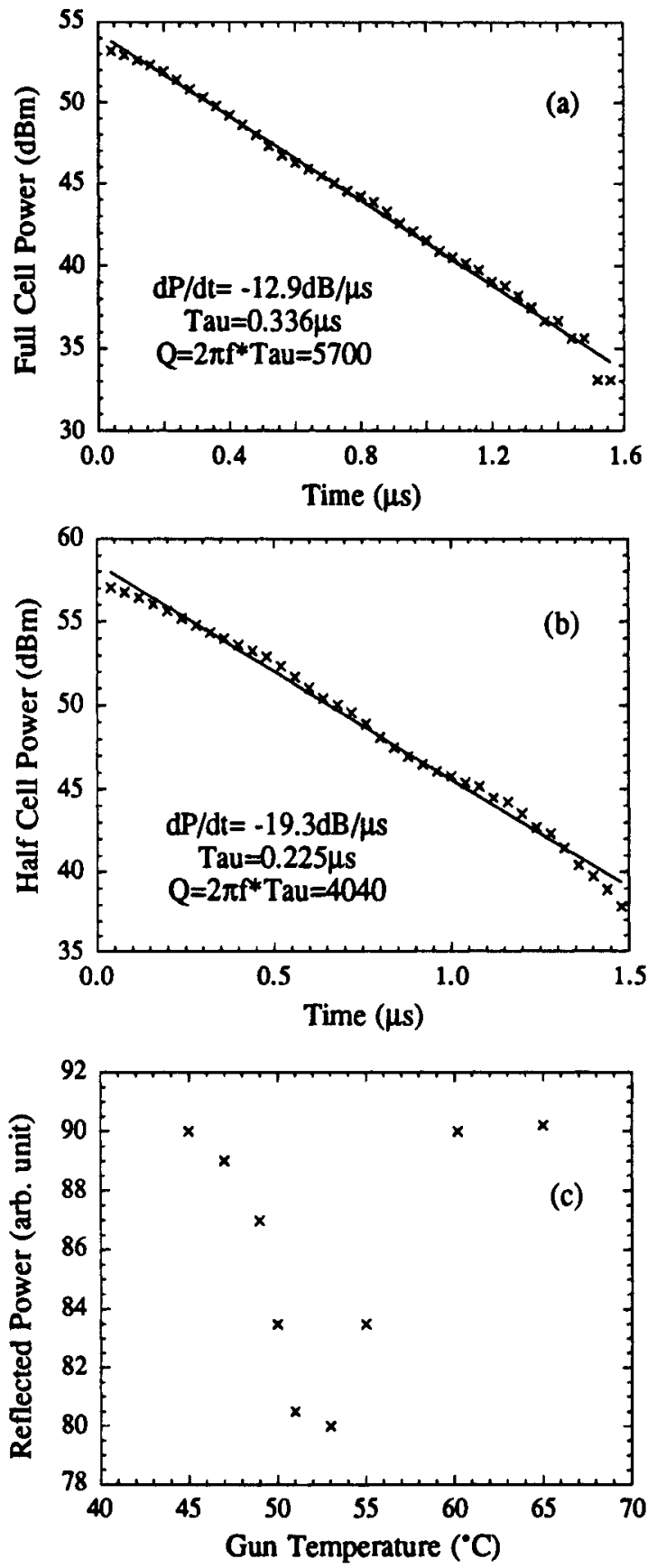

Figure 2: The $Q$ value measurements for full cell (top) and half cell (middle), and the if power reflected from the gun as a function of gun temperature (bottom). 
The beam leaves the laser with a linear polarization. Passing through a halfwave plate, the plane of polarization is rotated by an angle of $2 \theta$ if the plate is rotated by $\theta$. Here the quantity of interest is the projection of the wave electric field onto the surface of the cathode. The laser beam spot size on the cathode can be controlled by changing the focal length of the beam. The laser beam has Gaussian distribution in time and space.

While laser timing jitter has been minimized by adding a feedback stabilizer between the laser and mode-locker, the problem of amplitude fluctuation at the final UV output has not been rectified so far. Allowing the amplitude to fluctuate more than $50 \%$, the laser beam energy leaked at a prism is monitored by a photodiode at every shot. This photodiode was calibrated against a pyroelectric detector, since the latter needs too much of beam energy to be a useful online diagnostics. There is a linear correlation between the two. Other diagnostics include autocorrellator, spectrometer, and streak camera.

\section{RF SYSTEM AND CONTROL}

The RF system consists of low level RF driver, high power RF, and modulator[4] that drives the klystron. The operating frequency of $2.856 \mathrm{GHz}$ is derived from a crystal oscillator at $38.08 \mathrm{MHz}$ by using a 75 times multiplier. This multiplier is a frequency synthesizer based on a phase-locked loop(PLL). The mode locker provides the rf phase as a reference to the PLL. The PLL output frequency is down converted by a Mod- $\mathrm{N}$ counter. The counter output is phase compared with the reference, with the amplified and loop filtered difference driving a voltage controlled oscillator. The stability of this multiplier is as good as the mode locker, and the spectral purity is such that all the other frequencies are at least $70 \mathrm{~dB}$ down from the $2.856 \mathrm{GHz}$ center frequency. Concerning the amplitude, the biggest cause of variation is the ambient temperature. If the temperature is stable within $1^{\circ} \mathrm{C}$, the output power will remain constant within $0.25 \%$.

A coaxial phase shifter controls phasing of the laser pulse with respect to the rf phase, both of which are monitored on a digital sampling oscilloscope. This phase shifter is followed by a long cable to a pulsed preamplifier and a $1 \mathrm{~W} c \mathrm{cw}$ amplifier, which drives a number of beam position monitors (BPM)[10]. The ProComm preamplifier consists of one $30 \mathrm{~dB}$ gain solid state $\mathrm{cw}$ amplifier and three stages of $7 \mathrm{~dB}$ tuned-cavity triode amplifiers. This amplifier has a rise time of about $1 \mu \mathrm{s}$. In order to supply a flat top input signal to the klystron, the amplifier is turned on $2 \mu$ s earlier than the modulator trigger, and stays on for about $8 \mu \mathrm{s}$. When the drive amplifier is on, with the modulator not yet turned on, the absence of the electron beam in the klystron cavity leads to a gross missmatch of input to the klystron resulting in most of the input power being reflected. Subsequent turn on of the modulator reduces this reflection to less than $10 \%$. Monitoring of the forward and reverse signal between the pulsed amplifier offers an excellent diagnostics of both the low level system and the klystron amplifier. A number of isolators placed at various stages provide protection against excessive reflection of rf power to the previous stage. 
About 300 watts of pulsed rf power is fed into a SLAC XK- 5 klystron for up to $30 \mathrm{MW}$ of final rf power with $2.5 \mu \mathrm{s}$ pulse length. This power is divided evenly into two branches by a $3 \mathrm{~dB}$ coupler so that half of it drives the photoinjector through a variable attenuator and the other one half drives the PWT Linac $[6,7]$ through an attenuator and a phase shifter. The phase shifter sets the phase relationship between the photoinjector and the PWT linac. Since the acceleration gradient of both the gun and linac is proportional to the square root of the input rf power, stability of klystron output power is compelling in terms of reproducibility for the control of beam energy. With the input rf power being fairly stable in frequency, phase, and amplitude, the dc power from the modulator needs to be controlled in such a manner that the shot to shot variation is minimal.

Timing jitter of the modulator thyratron is less than a few tens of nanoseconds and is not a problem. The pulse forming network (PFN) consists of $N=20$ stages of $C=14 \mathrm{nF}$ capacitors and $L=0.686 \mu \mathrm{H}$ inductors. The characteristic impedance of the PFN is $Z_{0}=\sqrt{L / C}=7.0 \Omega$ with a pulse length of $\tau=2 N \sqrt{L C}=$ $3.92 \mu \mathrm{s}$. Variable inductors are made of 0.375 inch od copper tubing wound to form a multi-turn, 5 inch od helix. Each capacitor with a $50 \mathrm{kV}$ rating has a capacitance variation less than a few percent from specified value. Each tapped inductor is adjusted in such a way that the resonance frequency $\omega=\omega_{0} / \alpha$, where $\omega_{0} / 2 \pi=1.624 \mathrm{MHz}, \omega_{0}=1 / \sqrt{(0.686 \mu H)(14.0 n F)}$, and the actual capacitance $C=(14.0 \alpha) \mathrm{nF}$. After this is done for each stage, the PFN is charged up to $5 \mathrm{~V}$ and, in place of a thyratron, a switching transistor is triggered at $20 \mathrm{~Hz}$ or higher to dump the PFN energy to a $7.00 \Omega, 0.25$ watt resistor. For a high power test of the modulator, a pair of copper plate electrodes immersed in a tank full of cupric sulfate solution makes a high power resistor. The voltage waveform across that 7 $\Omega$ load is monitored while further adjustment of inductance values are made, to produce a flat top pulse at the load. This is to match the impedance of each stage and of overall PFN to that of klystron's pulse transformer primary winding. Since the cathode is pulsed at about $300 \mathrm{kV}$, a $1 \%$ of cathode voltage variation results in $1.5^{\circ}$ of rf phase change. For optimal rf waveform out of klystron, a feed-forward system[5] may be introduced at the low level side to minimize ripple in phase and amplitude.

Following the PFN tuning, there is still one more problem to solve: The AC line voltage needs to be regulated. Typically, the three phase $480 \mathrm{~V}$ line voltage is lowered by a ganged variac, then stepped up and full wave rectified to produce up to $50 \mathrm{kV}$ of dc, which is the PFN voltage. This line voltage fluctuates by as much as $\pm 5 \%$. The PFN voltage regulation is done as follows. A voltage divider measures the PFN voltage in real time to register 1 volt per $10 \mathrm{kV}$. A multi-turn potentiometer sets a reference voltage derived from a precision voltage reference. The difference is amplified, dc level shifted, and controls an SCR gate driver by feedback controlling the conduction angle of each of six SCR arrays. In this way, any overshoot or repetition rate dependence of the PFN voltage is avoided. The SCR gate driver is available commercially, and the PFN voltage regulation is better than $0.1 \%$ of the set voltage. 
Other than voltage control by variacs, there is no active regulation of electric power to the klystron filament. Line voltage fluctuation, as simulated by the change in variac setting, within a few percent has no appreciable effect on the klystron output. The electron emission off the klystron cathode is not temperature limited, but it is space charge limited. Only the cathode voltage is an important parameter. The same is true for the thyratron. The heater and reservoir electric power, and fluctuation thereof, have little effect to the closing characteristics of the device in terms of switching time and impedance at conduction as far as the reservoir voltage is not excessively high to cause self triggering.

Square law crystal detectors are employed for rf power measurements at various stages of interest. Calibrations are made on each detector for output voltage versus rf power. Since detectors, as well as some thermistor probes of powermeters, are not $50 \Omega$ devices, higher voltage standing wave ratio (VSWR) can lead to erroneous results, unless enough attenuation is introduced to minimize the VSWR at the rf source. The output voltage $V_{d}$ of a crystal detector, when terminated by a $50 \Omega$ load, and the rf power $P_{\mu w}$ as seen by the detector have been found to have the following relationship. $P_{\mu w} / \mathrm{dBm}=\sum_{i=0}^{n} a_{i} X^{i}$, where $X=\log _{10}\left(V_{d} / \mathrm{mV}\right)$, and $a_{i}$ are the coefficient to be determined by a least square fit. Usually, $n=3$ is sufficient for the power ranging from $-10 \mathrm{dBm}$ to $15 \mathrm{dBm}$.

\section{BEAMLINE AND DIAGNOSTICS}

The beamline consists of the rf gun followed by a solenoid focusing magnet and a drift tube, laser coupling box, quadrupole triplet, a dipole bending magnet, and a beam dump, which also serves as a Faraday cup. The solenoid is matched with a bucking coil at the back side of the gun, to provide a field free region on the surface of the cathode. The solenoid can produce up to $3 \mathrm{kG}$ of axial field. There are a number of diagnostics placed along the beamline, which will be described later.

The entire assembly sits on two laser optics tables measuring 3 feet by 5 feet each.Each element has fiducial marks inscribed at the top and/or midplane. A transit pointed along the beam axis detects any misalignment. There are several six-way vacuum crosses to accommodate diagnostics and pumping ports. In line gate valves separate the beamline into sections so that venting during the installation or removal of control and diagnostics is localized.

The entire setup is covered by layers of lead bricks to provide radiation shielding. This shielding is sufficient for beam energies up to $5 \mathrm{MeV}$. However, for linac operation at energies of $\simeq 20 \mathrm{MeV}$, neutron generation becomes significant. It is therefore planned to build a concrete structure of three feet thickness and house the beamline inside, with lead bricks remaining for local shielding.

Measurements of $\mathrm{rf}$ and laser beam power have been described in the previous sections. At the beamline, short bunches of photoelectron beams out of the rf gun are focused by a solenoid. When they pass through the laser coupling box, the first diagnostic is a Cherenkov radiator mounted on a pneumatic actuator. The radiation is in visible range, and the image is captured by a streak camera to 
be stored for further processing. This is for bunch length measurements and the projection of the image on the $y=0$ plane shows the side view of the bunch. Streak camera measurements are currently underway.

Following the radiator is a phosphor screen for beam profile measurements. The phosphor layer is bound to an aluminum base plate by a chemical agent, and a rectangle is scribed on it so that, when viewed from a right angle to the beam axis, it appears to be a square, providing a reference length for beam size determination. The aluminum piece is a cylindrical object machined at $45^{\circ}$ to its axis. It is also mounted on a pneumatic actuator. By floating the aluminum, actuator, and flange electrically, this profile monitor also serves as a Faraday cup. A CCD camera is paired with each phosphor screen at the beamline.

As nondestructive diagnostics, beam position monitors(BPMs)[10] are placed adjacent to each phosphor screen. The probe part of the BPM consists of four copper strips running parallel to the beam inside a beam pipe, and the downstream end of each strip is terminated to the ground. These are basically pickups of the electric fields of the electron bunches. A hybrid produces sum and difference from a pair of strips, left/right or top/bottom. Through mixers, these signals are down converted by $2.856 \mathrm{GHz}$ to low frequencies, which are subsequently amplified and integrated. The sum represents the total bunch charge, whereas the difference indicates the transverse position of the bunch with respect to the geometric axis of the BPM. Steering magnets are used to match the beam axis to the BPM and the magnetic center of quadrupole magnets.

Faraday cups are placed at the ends of the beamline to measure the total charge of the beam: One is at the end of straight line, the other at the end of branched line after the dipole. In order to capture all the electrons at relativistic energies, a Faraday cup is made of an aluminum block in the shape of circular cylinder. The cylinder is two inches in height along the beam axis, and one inch in diameter. In its simplest form, a Faraday cup is a capacitor, which is charged up by the beam electrons. The capacitance is increased by a coaxial cable running from the cup to a scope. The $1 \mathrm{M} \Omega$ oscilloscope input impedance is a bleeder resistor from the circuit point of view. The charge $q$ collected by the cup is given by $q=C V$, where $V$ is the oscilloscope voltage. If the cable is terminated by a $50 \Omega$ load, the current $i$ is $i=V / 50$, which is useful only for a dark current measurement within a single rf pulse.

Another diagnostic device for the measurement of photoelectron charge is an integrating current transformer (ICT). It is a capacitively shorted transformer with a core made of thin ribbons of cobalt/molybdenum amorphous alloy. As the beam passes through the probe, the capacitor is charged up, followed by discharge through the primary winding. The secondary is terminated by a parallel connection of a capacitor and a $50 \Omega$ for readout. The response has time delay and stretching of the pulse length, but the amplitude of the response is proportional to the charge of the bunch, which may have a subpicosecond rise time. This probe has been cross calibrated with the BPM and Faraday cup, resulting in good linear relationship among them. This is a nonperturbing and independent source of data for the measurement of photoelectron beam bunch charge. Figure 3 shows 


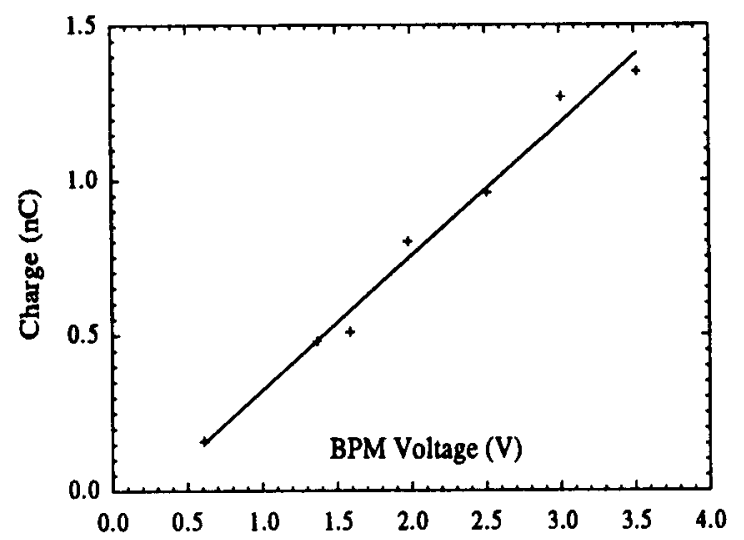

Figure 3: Calibration of the BPM using the ICT as a reference.

the bunch charge as measured by the ICT plotted against the BPM output.

\section{INITIAL ELECTRON BEAM MEASUREMENTS}

With the present system configuration[8], we can change the power level in the gun, and the rf phase with respect to the laser pulse, which set the cathode acceleration gradient. Temporal profile of the if pulse remains the same as long as the PFN tuning remains unchanged. All the other rf parameters such as coupling, frequency, and cavity $Q$ value are not subject to change during the photoelectron beam measurements.

Unlike the rf drive power, the laser beam parameters can be completely changed with relative ease. The angle of incidence, wave polarization, pulse length, and intensity have all been varied individually to study their effect on photoelectron generation. The solenoid focusing was extensively used to maximize the charge collection. In this section we report some of the results obtained at a pulse duration of $50 \mathrm{ps}$.

Given the large electric field present in the gun, an electron beam (dark current) is produced even when the cathode is not illuminated. Propagating the dark current through the beam line produces an energy spectrum as shown in Fig. 4. Each data point represents one rf pulse. Using a dipole magnet and a Faraday cup as a single channel spectrometer, the distribution would have been vertical somewhere between 2.9 and $3.1 \mathrm{MeV}$, if there was no shot to shot fluctuation of the rf power. The dark current increases strongly with rf power (Fig. 5). The temporal profile of the dark current depends on the rf power to the gun and so does the duration of it. For the case of $8.3 \mathrm{MW}, 24 \mathrm{nC}$ over $2 \mu \mathrm{s}$ corresponds to $12 \mathrm{~mA}$ of average current and the charge per rf period is only $4 \mathrm{pC}$, whereas the charge of a photoelectron bunch is about $1 \mathrm{nC}$ over a small fraction of an $\mathrm{rf}$ period.

Fig. 6 shows two images on phosphor screen captured in a sequence; one with a laser beam and one without. The first one has contribution from the dark 
640 Photoelectron Beams from the UCLA RF Gun

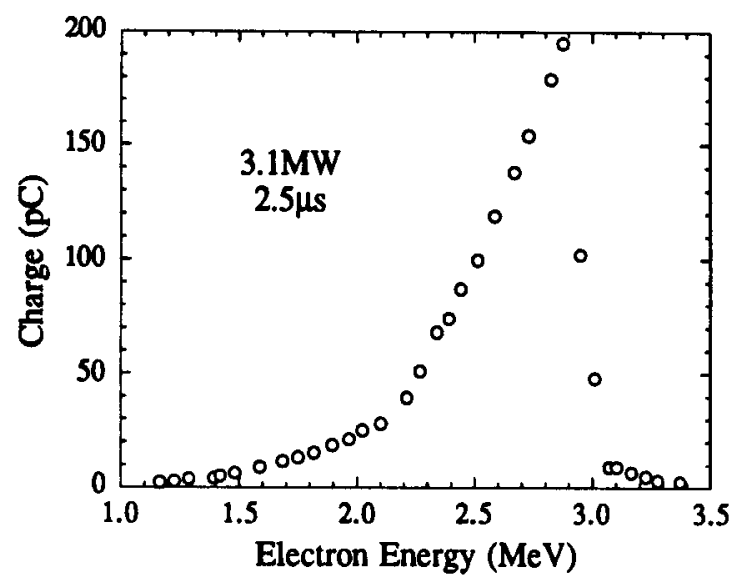

Figure 4: Energy spectrum of dark current.

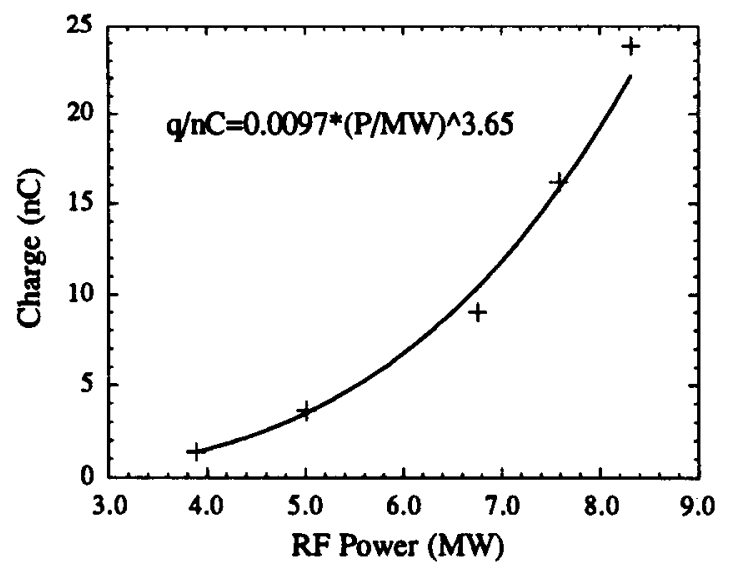

Figure 5: The rf power dependence of dark current. 

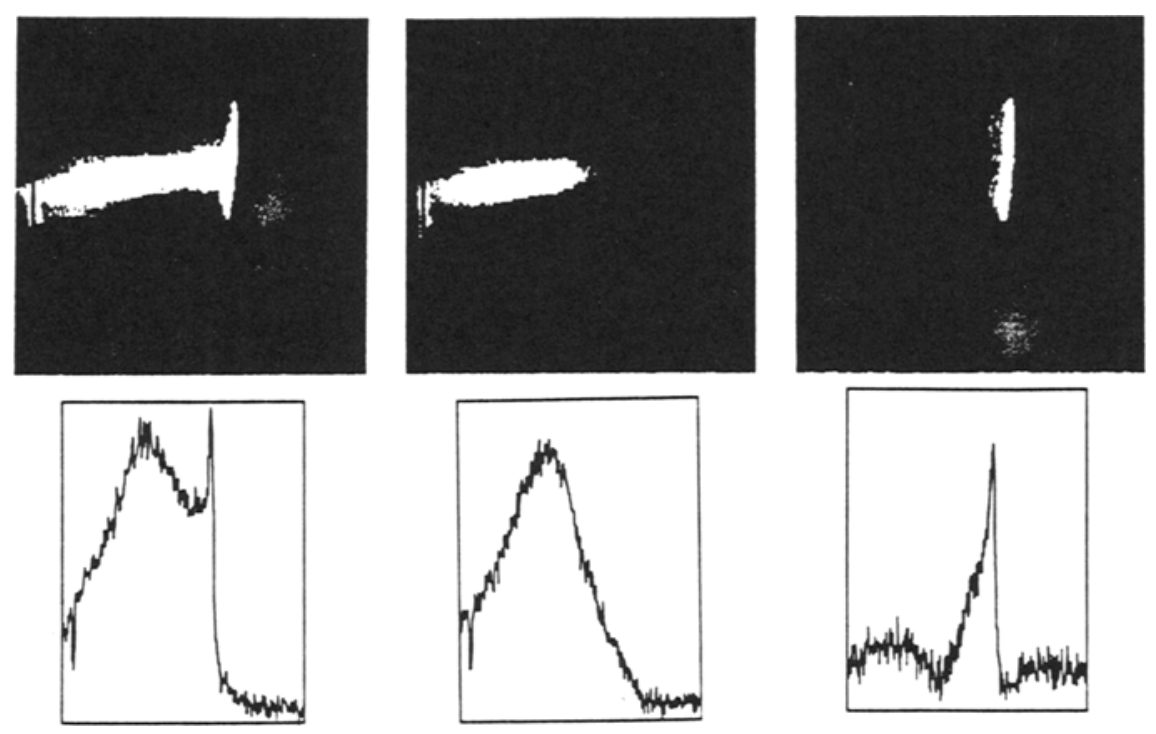

Figure 6: Image(top) and corresponding distribution(bottom) of photoelectron + dark current (left, $a$ ), dark current only (center, $b$ ), and photoelectrons (right, $c=a-b$ ). The horizontal axis represents energy dispersion of the beam.

current and the photocurrent, while the second has only dark current. This one is subtracted from the first to remove the effect of dark current. The horizontal full scale on each frame of Fig. 6 represents about $4 \%$ of momentum spread. The vertical axis of the distribution is proportional to the electron density in arbitrary units. A monochromatic beam would have resulted in a delta function distribution. For the frames of image, horizontal is momentum space and vertical is real space. The head and tail of this long pulse beam are subjected to a different $\mathrm{rf}$ phase and acceleration. The consequence of this is manifested in high emittance and larger momentum spread of $\Delta p / p \simeq 0.5 \%$.

A preliminary emittance measurement with the 50 ps long pulse was done to test the beam transport and diagnostic system. As shown in Fig. 1, the beam out of the gun passes through a quadrupole triplet, a dipole, and then makes a profile on a phosphor screen. The beam transport matrix elements are evaluated by using calibration data of dipole and quadrupole. The beam spot size is measured as a function of current through a quadrupole. A least square fit of this data according to the transport matrix provided a normalized emittance of $12 \pi$ $\mathrm{mm}$-mrad for this $50 \mathrm{ps}, 3.0 \mathrm{MeV}$ photoelectron beam as shown in Fig. 7.

For a given laser beam energy per pulse with the pulse length fixed, one effect of varying the spot size is to change the local power density, and the other is varying the area in which photoelectrons are borne. While electrical conductivity of copper is one of the highest among metals, a characteristic heat conduction time 


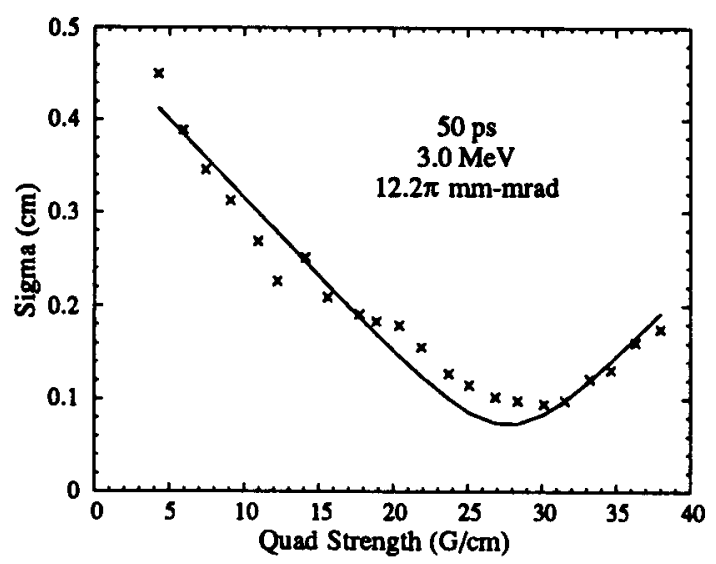

Figure 7: Emittance measurement of $50 \mathrm{ps}, 3.0 \mathrm{MeV}$ photoelectron beam.

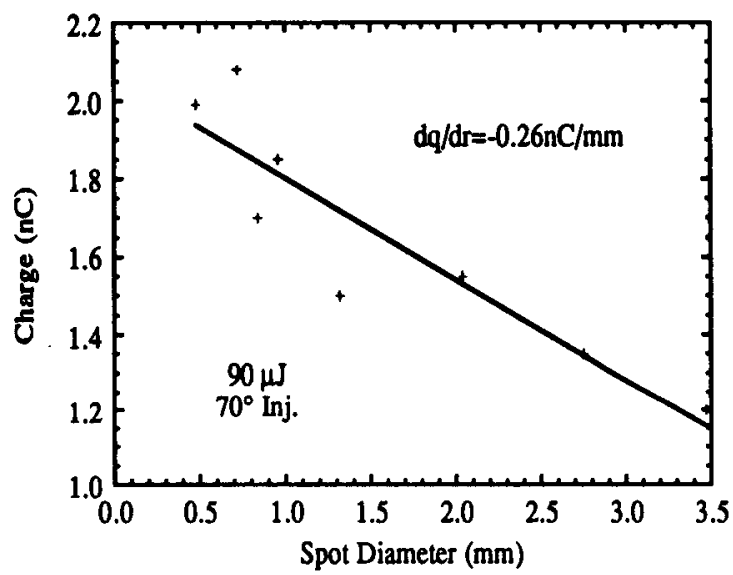

Figure 8: Dependence of photoelectric charge on laser beam spot size on the cathode. Laser energy was kept constant and the angle of incidence was $70^{\circ}$.

is not short enough to conduct away the heat generated on the cathode by laser beam of shorter than $1 \mathrm{~ns}$. Laser beams of pulse lengths much shorter than this time scale develop localized hot spot, which may be of thermionic significance. When the temperature of the lattice exceeds the melting point of copper, it is believed that the laser-rf combination initiates copper plasma that leads to an explosive emission. The probability of this event to occur begins to be appreciable for laser intensity of about $1 \mathrm{GW} / \mathrm{cm}^{2}$, according to the experiments done at Brookhaven[9].

Our measurements below this intensity limit are shown in Fig. 8. For a $70^{\circ}$ angle of incidence, the profile of the laser beam on cathode is elliptical, with its major axis in horizontal direction and the major diameter is $1 / \cos 70^{\circ}=2.92$ times the minor diameter. When the spot size is small, most of the particles are near the axis so that para-axial beam trajectories are ended at the Faraday cup, 
leading to higher collection efficiency and thus higher apparent quantum efficiency. As the spot size is made larger, more off-axis particles are lost before they reach the Faraday cup. In this case, a non-zero radial electric field of the rf waves would contribute to this process. Also, small size of the tubing between the window and cathode scrapes off some of the laser energy.

The present status and preliminary experimental results on quantum efficiency can be summarized as follows:

- The rf gun was driven by up to $8.3 \mathrm{MW}$ of $\mathrm{rf}$ power. Laser beam pulses of 50 ps, $266 \mathrm{~nm}$ with energies up to $100 \mu \mathrm{J}$ were used to generate photoelectron beams with up to $2 \mathrm{nC}$ of charge.

- At a given laser energy, more electrons are collected from a smaller spot size. But the efficiency is saturated.

- Preliminary quantum efficiency measurements indicate that the efficiency is that measured by other groups at $0^{\circ}$.

For lower energy spread within a bunch, shorter bunch length is favored. If the pulse length is too short, excessive intensity of the laser may result in explosive emissions and a damaged cathode. The apparent quantum efficiency is based on collected charge. Therefore, the collection efficiency must be understood in terms of beam dynamics, with emphasis on space charge effect and trajectories of off the axis particles. Also, microscopic studies of the cathode and measurements of its thermal response will lead to a better understanding of photoemission characteristics of the cathode.

\section{CONCLUSIONS}

We expect to compress the laser pulse to $4 \mathrm{ps}$ in the near future, and perform a complete series of measurements on quantum efficiency at $70^{\circ}$ and $2.5^{\circ}$, beam emittance, energy spread and pulse length. Once the gun is completely characterized we will accelerate the beam to $20 \mathrm{MeV}$ and start the experimental program on beam-plasma interaction and FEL.

The linac is expected to be operational in 1993. In the mean time, every effort will be made to achieve the best quality beam in terms of low emittance and high brightness. To that end, theoretical and experimental studies, along with reinforcement of experimental environment, will be continued.

\section{ACKNOWLEDGEMENT}

This work has been supported by US Department of Energy under Grant DEFG03-92ER-40493 and by Office of Naval Research through ONR-SDIO Grant \# N00014-90-J-1952. 


\section{REFERENCES}

[1] J.W. Dodd, et al., Proc. Part. Accel. Conf. (San Francisco, 1991) p. 2751

[2] P. Chen and J. M. Dawson, in Laser Acceleration of Particles, C. Joshi and T. Katsouleas, Eds., AIP Conf. Proc. No. 130, p. 201

[3] C. Pellegrini, in High Gain, High Power Free Electron Laser, R. Bonifacio, L. De Salvo Souza and C. Pellegrini, Eds., (Elsevier, 1989) Also, K.T. McDonald, IEEE Trans. Electron Dev. ED-35(1988) 2052.

[4] W. R. Bradford, et al., "Modulators," in The Stanford Two-Mile Accelerator, R. B. Neal, Ed., (Benjamin, New York, 1968) pp. 411-462

[5] I. Ben-Zvi, et al., Proc. Part. Accel. Conf. (San Francisco, 1991) p.1323

[6] D. A. Swenson, European Part. Accel. Conf. (Rome, 1988) p. 1418

[7] S. Hartman, et al, IEEE Part. Accel. Conf. (San Francisco, 1991) p. 2967

[8] S. Park, et al., Proc. 16th International Linac Conf (Ottawa, Canada, 1992)

[9] X.J. Wang, et al., J.Appl.Phys. 72, 888 (1992)

[10] J.T. Rogers et al.,, BNL Report, unpublished (August, 1991) 